\title{
Interspecific Interactions Affect Pests Differently
}

Audrey Errard, S. Baldermann, S. Kühne, I. Mewis, J. Peterkin and C. Ulrichs

\begin{abstract}
Spider mites, Tetranychus urticae Koch (Acari: Tetranychidae) and aphids, Myzus persicae (Sulzer) (Pterygota: Aphididae) share many host-plants, similar abiotic conditions and are world-wide distributed therefore, they often occur simultaneously in crops. However, the effects of interspecific interactions on the biology of these pests were poorly investigated. To test if they perform differently under intra- versus inter-specific interactions, host-plant acceptance, fecundity, survival, the total number of individuals and the rate of increase in the number of individuals were studied doing non-choice bioassays under laboratory conditions with leaf discs of tomato (Solanum lycopersicum L. 'Ailsa Craig'), pak choi (Brassica rapa L. var. chinensis 'Black Behi') and bean (Phaseolus vulgaris L. 'Saxa'). Alone, the pests differently accepted the host-plants. The acceptance of pak choi by spider mites was lower under interspecific interactions and higher on tomato for aphids. In general, spider mites' performance decreased when aphids were present; the fecundity, the number of individuals and the rate of increase being significantly lower on pak choi and bean. In contrast, aphids produced more offspring in the presence of spider mites, leading to a higher rate of increase in aphids individuals on tomato and pak choi. Thus, pest' responses to interspecific interactions is species-specific.
\end{abstract}

\section{Introduction}

Cultivated crops have to cope with various sources of disturbance from their abiotic and biotic environment. Thus, the effects of pest infestations on plant quality and yield have always received much interest. Phytophagous arthropods such as the spider mite, Tetranychus urticae Koch and the green peachaphid, Myzus persicae (Sulzer) have a short generation time leading to rapid and severe infestation in hundreds of crops worldwide. Besides, they have a similar host-plant range from various families including Rosaceae (rose, apple), Solanaceae (tomato, tobacco), Fabaceae (bean, pea) and Brassicaceae (pak choi). Therefore, crop species often have to defend against both pests in the field. It is therefore necessary to understand pest-pest interactions so as to better control them. Surprisingly, although the effects of abiotic factors on the life-cycle of spider mites and aphids alone on various plant species were investigated, little is known about the effects of biotic factors on these pest species.

In a general context, pest-pest interactions have received little attention as pointed out in the literature (Johnson 1990; Jaglan and Singh 2007). Studies on aphids mainly focused on transmittance of viral diseases (reviewed by Brault et al. 2010). Other studies assessing the interactions between spider mites and other pests such as thrips (Frankliniella occidentalis) were usually carried out in the context of tritrophic 
interactions. For instance, Messelink et al. (2010) showed that predatory mites were more effective against $T$. urticae when $F$. occidentalis was also present. Pesticide resistance and plant defense are the two other recurrent topics that are discussed together with aphid-spider mite interactions. Most of the literature on these species dating from the 1950s-1970s aimed at assessing the efficiency of broadspectrum pesticides such as demeton-methyl (Gould 1960) and chlorodimeform (Gemrich et al. 1976). More recently, the effects of plant-derived pesticides including plant essentials oils and natural botanical pesticides such as pyrethrin, extracted from the petals of Tanacetum cinerariaefolium (Pavela 2009) and piperidine alkaloids derived from Piper longum L. (Park et al. 2002) were measured against multipleinfestation with aphids and spider mites. At last, in the context of plant defense against herbivory, plant metabolites ranging from arthropod inducible proteins (Zhu-Salzman et al. 2008) to herbivore-induced plant volatiles (reviewed by Dicke et al. 2009) were discovered. Taken together, most of the studies were carried out at biochemical and molecular levels and focus on the effects on the biology of the plant. However, there is a need to understand the response of pests when interacting together, at the level of the organism to continue to improve pest control methods.

To test if the two-spotted spider mite (T. urticae) and the green peach-aphid (M. persicae) perform differently in case of intra- versus interspecific interactions, non-choice bioassays were carried out under laboratory conditions to compare host-plant acceptance by the pests and their performance (survival, fecundity, the total number of individuals and the rate of increase in the number of individuals) when these pests were alone (single-pest infestation) or under interspecific interactions (multiple-pest infestation) on three crops species of economic importance: tomatoes (Solanum lycopersicum L. 'Ailsa Craig'), beans (Phaseolus vulgaris) and pak choi (Brasica rapa var. chinensis).

\section{Material and Methods}

\section{Pest Species}

Two-spotted spider mites (T. urticae) and green peach-aphids (M. persicae (Sulzer)) were provided by Katz Biotech AG (Baruth, Germany) and reared on beans (Phaseolus. vulgaris L. 'Saxa') and pak choi (Brassica. rapa var. chinensis 'Black Behi') for several generations in greenhouses $\left(25 \pm 2{ }^{\circ} \mathrm{C}, 55 \pm 15 \% \mathrm{RH}, 16: 8 \mathrm{~h}\right.$ (light: dark)). Before conducting the experiments, spider mites and aphids were acclimated to the laboratory conditions $\left(22 \pm 1{ }^{\circ} \mathrm{C}, \mathrm{RH} 25 \pm 3 \%, 16\right.$ : $8 \mathrm{~h}$ (light: dark)) on these host-plants.

\section{Plant Material}

Tomato and pak choi were grown for 7 weeks under greenhouse conditions $\left(25 \pm 2{ }^{\circ} \mathrm{C}, 55 \pm 15 \% \mathrm{RH}, 16: 8 \mathrm{~h}\right.$ (light: dark)). Common beans were grown under the same conditions for 3 weeks. 


\section{Experimental Design and Data Analysis}

Under laboratory conditions $\left(22 \pm 1{ }^{\circ} \mathrm{C}\right.$, relative humidity (RH) $25 \pm 3 \%$, photoperiod 16: $8 \mathrm{~h}$ (light: dark)), non-choice tests were performed with spider mites and aphids when these pests were alone (referred to here as the single-pest infestation assay) or were together (multiple-pest infestation assay) on leaf discs (1.8 cm diameter) from bean, pak choi and tomato. The discs were excised from fresh, fully-expanded leaves, avoiding damaged plant parts and primary vein axil then placed individually underside up on top of agar discs (1.5\%; $1.8 \mathrm{~cm}$ diameter) to delay desiccation of the plant material. Each disc was placed in the centre of a round Petri dish $(9.5 \mathrm{~cm}$ diameter, $0.8 \mathrm{~cm}$ depth). Each round Petri dish was then placed inside a square Petri dish (12.5 cm length, $1.5 \mathrm{~cm}$ depth); the latter was filled with $15 \mathrm{~cm}^{3}$ of glycerin solution (85\%) and $15 \mathrm{~cm}^{3}$ of water as a barrier preventing spider mites and aphids from escaping. Furthermore, this prevented excessive relative humidity around the leaf discs to meet species requirements. There were 15 replicates $(N=15)$ in each assay ('single' and 'multiple') on each plant species. In the single-pest infestation assay, seven $T$. urticae females per leaf disc or seven M. persicae females per disc were dropped off with a fine brush. In the multiple-pest infestation assay, two leaf discs from the same host-plant were used so that food was not a limiting factor. The two discs were placed in contact to allow the pest to move from one to the other, in each Petri dish. The discs were then infested with seven spider mite females and seven aphid females randomly. To avoid starvation, one (single-pest infestation) or two (multiple-pest infestation) new fresh leaf disc(s) was/were added to each Petri dish every $50 \mathrm{~h}$ after infestation. Newly added discs were placed in contact with the old ones so that the pest individuals could move from the old discs to the new ones. As arthropods are sensitive to the variations of their abiotic environment, the Petri dishes were rerandomized every $24 \mathrm{~h}$ to offset the effects of possible gradients of temperature and humidity in the laboratory.

Host-plant acceptance was defined as the percentage of females for one given pest species that settled after $24 \mathrm{~h}$ (Yano et al. 1998). The pests' performance was studied in terms of fecundity, hatching success of the eggs (for spider mites only), survival, total number of individuals at the end of the experiment and the rate of increase in the number of individuals over 6 days. Fecundity was calculated as the mean number of eggs per spider mite or offspring per aphid female per 5 days (Greco et al. 2006). Although individuals could return to the leaf discs after wandering in the round Petri dish, the glycerol barrier in the square Petri dish trapped the wandering individuals. Besides, dead individuals were removed from the leaf discs every $24 \mathrm{~h}$. Calculating mortality would, therefore, have introduced bias. Therefore, survival (rather than mortality) and fecundity were chosen as indicators of host-plant suitability. Survival was calculated according to Eq. 1, where $N$ refers to the number of females alive at the beginning of the experiment $\left(t_{0}\right)$ or still alive at a defined counting period ( $t_{n}$; where $n$ refers to the number of days after the beginning of the experiment). It was of 5 days for spider mites. After 3 days, new offspring of aphids appeared. From this point on it became impossible to distinguish between the newly formed adults from the individuals placed at the beginning of 
the experiment. To prevent a biased survival calculation, only values from the infestation time to 3 days backwards were taken into account.

Survival $=1-\frac{\mathrm{Nt}_{0}-\mathrm{Nt}_{\mathrm{n}}}{\mathrm{Nt}_{0}}$

The eggs of the spider mites hatched after 6 days. The hatching success of the eggs was calculated as the ratio between the number of offspring after 6 days and the number of eggs laid after $24 \mathrm{~h}$. The total number of individuals per Petri dish was counted every 24 h over 6 days. The rate of increase (Eq. 2) was used as an indicator of the population growth. It refers to the variation in the mean number of individuals at a given counting time (t) over a given time period $(N) ; 6$ days in our study. It is calculated based on the ratio between the number of individuals $(A)$ at a given counting period (day 1-6) and the number of individuals placed at the beginning of the experiment $\left(t_{0}\right)$. The spider mites' offspring start to feed directly after hatching. Reproduction of aphids is viviparous and parthenogenetic and the first instar nymphs are also feeding. The $A$ values therefore include both the adults and the newly emerged individuals (for spider mites) and offspring (for aphids). $A$ values exclude the number of eggs of spider mites.

Rate of increase $\left(t_{0}, t\right)=\left(\frac{A(t)}{A\left(t_{0}\right)}\right)^{1 / N-1}$

The data were analyzed by Mann-Whitney $U$ tests and Kruskal-Wallis with SPSS 21 software because of heteroscedasticity of the data sets.

\section{Results}

\section{Host-Plant Acceptance}

The results showed that spider mites and aphids accepted differently the different host-plants in the two assays (Fig. 1a and Fig. 1b). The spider mites (Fig. 1a) accepted bean significantly better than tomato in the single-pest infestation assay. However, there were no significant differences between tomato and pak choi, and between pak choi and bean $(P \leq 0.05)$. In the multiple-pest infestation assay, tomato and bean were accepted significantly better than pak choi by spider mites $P \leq 0.05)$. No significant differences in acceptance were observed between tomato and bean $(P \leq 0.05)$. When comparing single versus multiplepest infestation for one given host-plant, the presence of aphids possibly reduced the acceptance of bean by spider mites, and positively influenced it on tomato, but the statistical analysis revealed no significant difference in either case $(P \leq 0.05)$. On pak choi, however, the host-plant acceptance by spider mites was significantly lower in the presence of aphids in comparison with the single-pest assay, when spider mites were alone $(P \leq 0.05)$ (Fig. $1 \mathrm{~A})$. 


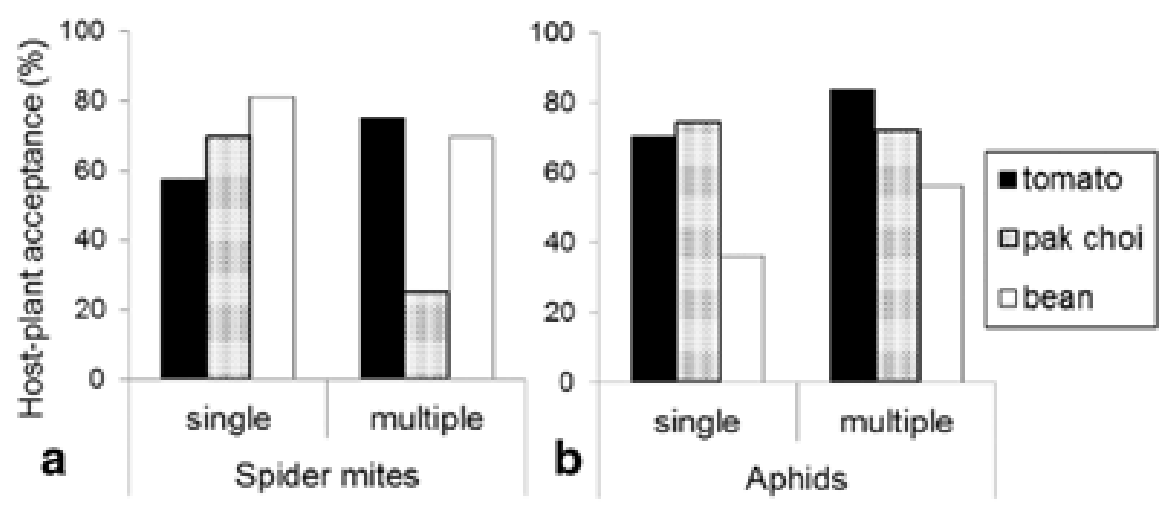

Fig. 1

Host-plant acceptance after $24 \mathrm{~h}$ by Tetranychus urticae (a) and Myzus persicae (b) when alone (single-pest infestation) and under interspecific interactions (multiple-pest infestation) on tomato, pak choi, and bean $(N=15)$. Significant differences between the host-plants within one assay are indicated with different letters. $(*)$ indicates that the multiple-pest infestation assay differs significantly from the single-pest assay for indicated host-plant species $(P \leq 0.05)$

When aphids were alone, they accepted tomato and pak choi significantly better than bean $(P \leq 0.05)$. No significant differences were observed between tomato and pak choi $(P \leq 0.05)$ (Fig. 1B). In the multiple-pest infestation assay, the aphids accepted tomato significantly better than bean. No significant differences were observed between tomato and pak choi nor between pak choi and bean $(P \leq 0.05)$. When comparing single versus multiple-pest infestation, more aphid females settled on tomato when spider mites were present (Fig. 1B). A similar trend, though not significant, was also observed on single vs. multiple infested beans ( $P \leq 0.05$ respectively). Host-plant acceptance by aphids did not differ significantly on pak choi in the multiple-pest infestation assay (Fig. 1B). To summarize, on two out of the three host-plants, acceptance by spider mites tended to decrease under interspecific interactions whereas acceptance by aphids tended to increase under specific interactions.

\section{Performance of the Pest Species}

Spider mites and aphids responded differently to interspecific interactions. For spider mites, fecundity, hatching success, survival, the number of individuals feeding and the mean rate of increase were significantly greater on beans than on pak choi and tomato in both assays ('single' and 'multiple')

(Table 1 and Fig. 2). When comparing single versus multiple-pest infestation, no significant differences were observed in the fecundity on tomato. On pak choi and beans however, the fecundity of the spider mites was significantly lower in the presence of aphids $(P \leq 0.05)$. Hatching success of spider mites' eggs was lower in the presence of aphids on tomato $(P \leq 0.05)$ and although not significantly, tended to be also lower on beans $(P \geq 0.05)$. Based on the correlation coefficients, the trends in the survival of adult spider mites switched between the single and the multiple-pest infestation assays (Fig. 2). The survival of spider mites on tomato followed an exponential decay $\left(y=a \cdot e^{-b x}\right)$ when spider mites were alone $\left(R^{2} s=0.97\right)$ and a linear one $\left(R^{2}{ }_{M}=0.978\right)$ when aphids were present $(y=-a x+b)(F i g$. 2A). At the end of the experiment, the 
relative survival rate of spider mites was significantly higher on tomato in the presence of aphids than in the single-pest infestation assay $(P \leq 0.05)$. On pak choi (Fig. $2 C$ ), the survival of the spider mites followed an exponential pattern of decrease in the multiple-pest infestation assay and a linear function when the females lived alone on the discs. However, at the end of the experiment, the survival of spider mites on pak choi was not significantly different between the two assays. It is therefore likely that interspecific interactions did not affect significantly the survival of adult spider mites on this host-plant. On beans, the survival of spider mites followed an exponential pattern of decrease in the multiple-pest infestation assay, whereas it decreased linearly and was significantly lower without aphids ( $P \leq 0.05)$ (Fig. 2E). Regarding the number of individuals feeding, there were significantly more spider mites when kept alone on pak choi, than in the presence of aphids $(P \leq 0.05)$. These results are consistent with the rate of increase observed which was much greater on pak choi in the single-pest infestation. On bean, there were about $40 \%$ more spider mites for the single-pest infestation assay than in the case of the multiple-pest infestation $(P \leq 0.05)$ and the total number of spider mites feeding increased at a higher rate in the single-pest assay (Table 1). Taken together these results indicated that the presence of aphids had a negative impact on the spider mites. Although no significant differences were found for the number of mites feeding and the mean rate of increase on tomato, similar decreases under specific interactions for those variables were observed as on pak choi and bean. To summarize, interspecific interactions impacted negatively the performance of spider mites. 
Table 1

Species' performance on the different host-plants when alone (single) or under interspecific interactions (multiple)

\begin{tabular}{|c|c|c|c|c|c|c|c|c|c|c|c|c|}
\hline & \multicolumn{5}{|c|}{ Spider mites } & \multicolumn{7}{|c|}{ Aphids } \\
\hline & \multicolumn{3}{|l|}{ single } & \multicolumn{2}{|l|}{ multiple } & \multicolumn{4}{|l|}{ single } & \multicolumn{3}{|l|}{ multiple } \\
\hline & Tomato & Pak choi & Bean & Tomato & Pak choi & Bean & Tomato & Pak choi & Bean & Tomato & Pak choi & Bean \\
\hline \multirow[t]{2}{*}{ Fecundity } & $5.6 \mathrm{a}$ & $10.5 \mathrm{~b}$ & 27.9 & 7.9 & $1.9 \mathrm{~B}^{*}$ & 15.8 & $0.9 a$ & $0.6 a b$ & 0.0 & 4.3 & $1.4 \mathrm{~B}^{*}$ & 0.0 \\
\hline & & & c & $A$ & & $\mathrm{C}^{*}$ & & & $\mathrm{~b}$ & $A^{*}$ & & $C$ \\
\hline \multirow{2}{*}{$\begin{array}{l}\text { Hatching } \\
\text { success }\end{array}$} & $36.1 \mathrm{a}$ & $36.0 \mathrm{a}$ & 67.0 & $16.3 \mathrm{~A}$ & 39.5 & 62.3 & - & - & - & - & - & - \\
\hline & & & $\mathrm{b}$ & $*$ & $A B$ & B & & & & & & \\
\hline \multirow{2}{*}{$\begin{array}{l}\text { Number of } \\
\text { individuals } \\
\text { feeding }\end{array}$} & $7.7 \mathrm{a}$ & $13.0 \mathrm{a}$ & 45.9 & 2.9 & 4.3 & 27.5 & $10.6 \mathrm{a}$ & 8.8 & 0.3 & 48.5 & $17.0 \mathrm{~B}^{*}$ & 0.0 \\
\hline & & & $\mathrm{b}$ & $A$ & $B *$ & $\mathrm{C}^{*}$ & & $\mathrm{a}$ & $\mathrm{b}$ & $A^{*}$ & & $C$ \\
\hline \multirow{2}{*}{$\begin{array}{l}\text { Mean rate } \\
\text { of increase }\end{array}$} & $1.5 \mathrm{a}$ & $10.9 \mathrm{a}$ & 36.8 & -13.5 & -7.7 & 25.6 & $7.2 \mathrm{a}$ & 4.8 & -39 & 38.1 & 15.9 & $-100 C$ \\
\hline & & & $\mathrm{b}$ & A & $A^{*}$ & $\mathrm{~B}^{*}$ & & $a$ & $8 b$ & $A^{*}$ & $\mathrm{~B}^{*}$ & \\
\hline
\end{tabular}

Different letters indicate significant differences between the host-plants for one given assay ('single' or 'multiple'); Mann-Whitney U tests

The statistical analysis on the hatching success and the population rate of increase was performed on $\arcsin (\mathrm{V})$ transformed data and expressed in the table as percentages

*Indicates significant differences between single versus multiple-pest-infestation $(P \leq 0.05)$; Kruskal-Wallis tests 

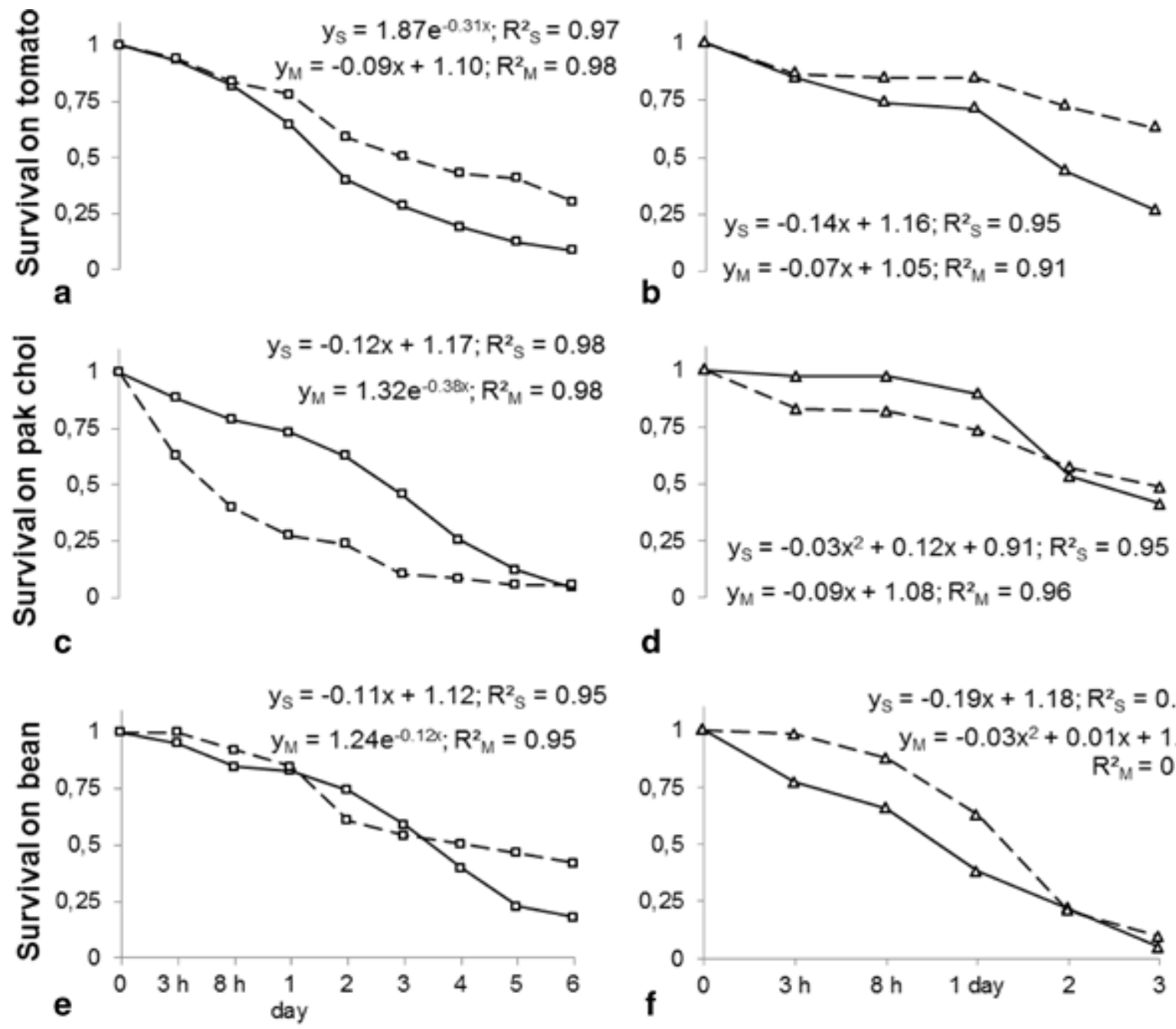

Spider mites

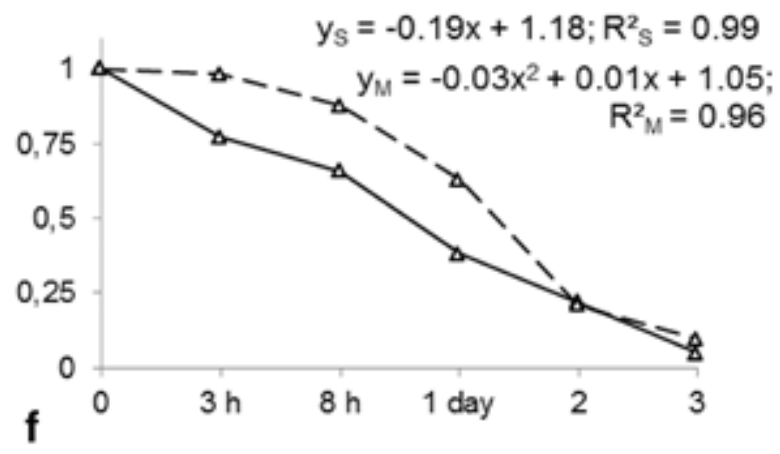

Aphids

Fig. 2

Survival of spider mites and aphids over time on tomato $(\mathbf{a}, \mathbf{b})$, pak choi $(\mathbf{c}, \mathbf{d})$ and bean $(\mathbf{e}, \mathbf{f})$ in the singlepest (solid line) and multiple-pest infestation (dashed line) bioassays. Correlation coefficient: $R^{2} ; S=$ singlepest infestation; $M=$ multiple-pest infestation; $N=15$

Aphids performed significantly better on tomato than on pak choi and bean in both assays (Table 1 ). The nutritional or biochemical effects of the food plant, rather than interspecific interactions, were certainly responsible for the results on beans. It was indeed observed that aphids turned to a brownish colour shortly after feeding on beans and died in the next $48 \mathrm{~h}$ regardless of the type of infestation. As a consequence the number of aphids feeding dropped to zero; the fecundity could not be assessed on the host-plant $P$. vulgaris. On tomato and pak choi however, when comparing single versus multiple-pest infestation, the fecundity of aphids was significantly greater in the presence of spider mites $(P \leq 0.05)$. On tomato (Fig. 2B), survival decreased linearly in both assays but to a greater extent in the case of single-pest infestation $\left(a_{s}=-0.14\right.$ vs. $\left.a_{M}=-0.07\right)$. At the end of the experiment, more aphids had survived when spider mites were also present on the leaf discs; than in the case of single-pest infestation. On pak choi (Fig. 2D) and bean (Fig. 2F) the best-fitting tendency curves varied between the single and the multiple-pest 
infestation assay. To summarize, although not significantly different, aphid survival tended to be greater on tomato, pak choi and beans under interspecific interactions (Figs. 2B, 2D, 2F). Thus, interspecific interactions with spider mites tended to increase aphids' fitness as higher values were observed in any treatment involving multiple-pest infestation. The number of aphids feeding and the rate of increase support this conjecture. When comparing single versus multiple-pest infestations, the number of aphids feeding on tomato was significantly greater in the presence of spider mites $(P \leq 0.05)$ (Table 1$)$. On pak choi, the number of individuals feeding was also nearly twice as high in the presence of spider mites as in the single-pest assay $(P \leq 0.05)$. To finish, on tomato and pak choi, the rate of increase was at least four times as high in the presence of spider mites than in the single-pest infestation $(P \leq 0.05)$ (Table 1$)$. Thus, interspecific interactions influenced positively the performance of aphids.

\section{Discussion}

Prior work has mainly documented the effects of abiotic parameters such as temperature and humidity on pests' biology. However, little attention was paid to the biotic factors such as interspecific interactions. To our knowledge, this is the first study assessing the effects of spider mite-aphid interactions on the hostplant acceptance by the pests and pest species' performance. The results showed that the pests accepted and performed on the host-plants differently. Common bean ( $P$. vulgaris) was the most suitable host for spider mites, a fact which was not surprising as the spider mites were reared on this plant species (Agrawal et al. 2002). Aphids, however, better accepted pak choi and tomato when compared with beans. Firstly, it is known that many species require time to adapt to another source of food (Egas et al. 2003) which explains on the one hand that acceptance of the different plant-species differed between the two pests in this study. On the other hand, biochemical properties of the host-plants can influence host-plant acceptance and pests' performance. It has indeed long been accepted that host-plant suitability regardless of the pest preference (not assessed in this study) can depend on the nutritional value of the food source; for instance, spider mites and aphids better accept and/or perform on plants with higher nitrogen and amino acid contents (reviewed by Wermelinger et al. 1991 for spider mites, reviewed by van Emden et al. 1969 for aphids). In the present study, the aphids placed on beans turned to a brownish colour shortly after feeding in both the single- and the multiple-pest infestation assays, indicating that bean is not a suitable food source for M. persicae. This assumption is also reflected by the shorter lifespan of aphids on beans compared with the other host-plants. It has been shown that $P$. vulgaris contains compounds, such as lectins, not digestible by, or toxic to aphids. These compounds are also present in jack beans (Canavalia spp.) and potatoes (Solanum tuberosum) because they have negative effects on population growth and development time of $M$. persicae and they contribute to the reduction of the palatability of the food due to their amylase inhibitor activity (reviewed by Van Damme 2008). Thirdly, wounding induces local plant defences which often involve the release of pest-repellent compounds. With regards to fecundity and hatching success, it has been shown for instance, that terpenoids released by tomato plants 
significantly enhanced egg mortality and delayed embryonic development of spider mites (Ament et al. 2004). Similarly, components in aphids' saliva can elicit local responses that in return impact aphids' fecundity (De Vos and Jander 2009). Khan et al. (2010) demonstrated an increase in the glucosinolate levels due to $M$. persicae feeding on Brassica crops. It is possible that these defense compounds can also affect food acceptance by, and fecundity of spider mites. Finally, leaf structures (e. g. trichomes) and texture (e.g. waxy surface) can influence host-plant suitability by affecting, for instance, the survival of aphids (Leite et al. 1999) and the feeding and oviposition of spider mites (Wilson 1994).

When comparing the single- versus multiple-pest infestation assays, the response of the pests changed on the host-plants under interspecific interactions. We found that the acceptance of pak choi by spider mites was lower in the presence of aphids. A similar trend was observed on bean. As host-plant acceptance was defined as the number of females that settled after $24 \mathrm{~h}$, the lower the host-plant acceptance, the larger the proportion of wandering individuals after $24 \mathrm{~h}$. Therefore, our results indicate that the presence of another pest species is likely to impede spider mite females in finding an appropriate place to settle by extending for instance, the searching time period before oviposition and/or adding more difficulties to the process of finding an appropriate place to lay eggs. This would therefore impact negatively the fecundity directly and the number of feeding individuals indirectly as observed on pak choi and bean. Taken together, the results suggest that, regardless of the suitability of the host-plant, interspecific interactions can affect not only the settlement but also the performance of spider mite females on their host-plant. Nevertheless, because the egg-hatching success did not differ significantly on these host-plants it can be concluded that the interference was not mediated through the development of the eggs. Therefore, the differences in the response of the spider mites to intra-versus inter-specific interactions may rather be the result of changes in their behavior (e.g. wandering activity) when aphids were also present. The shifts in the trends of the tendency curves of the survival of the females are in agreement with this conjecture as the trends differed between the single and the multiple-pest assays. Further investigations are necessary to provide information about the nature and mechanism of the effect(s) of interspecific interactions on survival. In contrast, the acceptance of tomato by aphids was significantly higher under interspecific interactions. Also the aphids performed better on tomato and pak choi under interspecific interactions. The results (significantly higher fecundity, number of individuals and rate of increase) propound that the presence of another pest under natural conditions can influence the aphid's population dynamics and their reproductive fitness. It is possible that aphids would invest more energy in reproduction to cope with interspecific interactions. Regarding specific properties of the host-plants, it is here also likely that the tested host-plants provided different local defense responses. For instance, it is known that the blend of volatiles emitted by the plants varies consequently under single- versus multiple-pest infestation (Moayeri et al. 2007; Dicke et al. 2009). Such responses would therefore differentially affect the spider mites and the aphids under interspecific interactions, and indirectly influenced the reproductive and survival of one as 
compared with the other pest. Thus, the different responses of the pests to the different host-plants may be the result of the combined effects of the pest's life-history and of the host-plant's physical and biochemical properties. In summary, the results showed that on the one hand spider mites and aphids responded differently to intra- versus interspecific interactions suggesting different behavioral responses of these two pest species to cope with interspecific interactions. As a consequence, the response to interspecific interactions is species-specific. On the other hand, it is interesting to note that similar trends in the general response of the pests to interspecific interactions were observed on at least two of the three host-plants tested for each pest species. This study therefore suggests that under suitable abiotic conditions (e.g. optimum temperature and relative humidity), there may be a recognizable pattern in the overall response given by one species to interspecific interactions regardless of the host-plant. In other words, one given species would respond similarly to interspecific interactions on different host-plants. Consequently, similar pest-management strategies could be applied to different crop species in monocultures. However, in the context of sustainable crop management involving a bigger diversity in the plant species at the same location, assessing host-plant preference would provide hints on the distribution of these two species in a complex cropping system. Eventually, more investigations are required to determine to what extent environmental conditions and the quality of the food can influence pest-pest interactions. As proposed by the stress-gradient hypothesis (SGH), negative interactions such as competition that are strong in appropriate climatic conditions would weaken across an increasing abiotic stress gradient (Bertness and Callaway 1994). Recent work has confirmed the predictions of the SGH for some insect herbivores (Dangles et al. 2013). Future work assessing life-history and life-table parameters of spider mites and aphids under harsher environmental conditions should therefore elicit further useful information on pest-pest interactions.

\section{References}

Agrawal AA, Vala F, Sabelis MW (2002) Induction of preference and performance after acclimation to novel hosts in a phytophageous spider mite: adaptive plasticity? Am Nat 159:553-565

Ament K, Kant MR, Sabelis MW, Haring MA, Schuurink RC (2004) Jasmonic acid is a key regulator of spider mite-induced volatile terpenoid and methyl salicylate emission in tomato. Plant Physiol 135:2025-2037 Bertness M, Callaway RM (1994) Positive interactions in communities. Trends Ecol Evol 9:191-193 Brault V, Uzest M, Monsion B, Jacquot E, Blanc S (2010) Aphids as transport devices for plant viruses. Compt Rend Biol 333:524-538

Dangles O, Herrera M, Anthelme F (2013) Experimental support of the stress-gradient hypothesis in herbivore-herbivore interactions. New Phytol 197:405-408 
De Vos M, Jander G (2009) Myzus persicae (green peach aphid) salivary components induce defence responses in Arabidopsis thaliana. Plant Cell Environ 32:1548-1560

Dicke M, van Loon JJA, Soler R (2009) Chemical complexity of volatiles from plants induced by multiple attack. Nat Chem Biol 5:317-324

Egas M, Norde D-J, Sabelis MW (2003) Adaptive learning in arthropods: spider mites learn to distinguish food quality. Exp Appl Acarol 30:233-247

Gemrich EG II, Lee BL, Tripp ML, Vandestreek E (1976) Relationship between formamidine structure and insecticidal, miticidal, and ovicidal activity. J Econom Entomol 69:301-306

Gould HJ (1960) Control of aphids and red spider mites on young carnations under glass. Plant Path 9:106109

Greco NM, Pereyra PC, Guillade A (2006) Host-plant acceptance and performance of Tetranychus urticae (Acari, Tetranychidae). J Appl Entomol 130:32-36

Jaglan RS, Singh R (2007) History of integrated pest management. In: Jain PC, Bhargava MC (eds)

Entomology: novel approaches. New Indian Agency Policy, New Delhi, pp. 1-18

Johnson KB (1990) Assessing multiple pest populations and their effects on crop yield. In: Teng PS (ed) Crop loss assessment in rice. International Rice Research Institute IRRI, Manila, pp. 203-213

Khan MAM, Ulrichs C, Mewis I (2010) Influence of water stress on the glucosinolate profile of Brassica oleracea var. italica and the performance of Brevicoryne brassicae and Myzus persicae. Entomol Exp Appl $137: 229-236$

Leite GLD, Picanco M, Guedes RNC, Skowronski L (1999) Effect of fertilization levels, age and canopy height of Lycopersicon hirsutum on the resistance to Myzus persicae. Entomologia Experimen Appl 91:267-273 Messelink GJ, Van Maanen R, Van Holstein-Saj R, Sabelis MW, Janssen A (2010) Myzus persicae (green peach aphid) salivary components induce defence responses in Arabidopsis thaliana. Biocontrol 55:387398

Moayeri HRS, Ashouri A, Poll L, Enkegaard A (2007) Olfactory response of a predatory mirid to herbivore induced plant volatiles: multiple herbivory vs. single herbivory. J Appl Entomol 131:326-332

Park B-S, Lee S-E, Choi W-S, Jeong C-Y, Song C, Cho K-Y (2002) Insecticidal and acaricidal activity of pipernonaline and piperoctadecalidine derived from dried fruits of Piper longum L. Crop Prot 21:249-251 Pavela R (2009) Effectiveness of some botanical insecticides against Spodoptera littoralis Boisduval (Lepidoptera: noctudiae), Myzus persicae Sulzer (Hemiptera: aphididae) and Tetranychus urticae Koch (Acari: tetranychidae). Plant Prot Sci 45:161-167 
Van Damme JM (2008) Plant lectins as part of the plant defence system against insects. In: Schaller A (ed) Induced plant resistance to herbivory, Springer Verlag, Heidelberg: pp 285-307

Van Emden HF, Eastop VF, Hughes RD, Way MJ (1969) The ecology of Myzus persicae. Ann Rev Entomol $14: 197-270$

Wermelinger B, Oertli JJ, Baumgartner J (1991) Environmental-factors affecting the life-tables of Tetranychus-urticae (Acari: tetranychidae). III. Host-plant Nutrition. J Experim Appl Acarol 12:259-274

Wilson L (1994) Resistance of okra-leaf cotton genotypes to twospotted spider mites (Acari: tetranychidae). J Econom Entomol 6:1726-1735

Yano S, Wakabayashi M, Tabakayashi J, Takafuji A (1998) Factors determining the host plant range of the phytophagous mite, Tetranychus urticae (Acari: tetranychidae): a method for quantifying host plant acceptance. Exp Applied Acarology 22:595-601

Zhu-Salzman K, Luthe DS, Felton GW (2008) Arthropod-inducible proteins: broad spectrum defenses against multiple herbivores. Plant Physiol 146:852-858 